Echeverría Samanes, B. y Martínez Clares, P. (2021). Hacia un ecosistema de investigación sobre formación profesional en España. Revista de Investigación Educativa, 39(1), 249-264.

DOI: http://dx.doi.org/10.6018/rie.424901

\title{
Hacia un ecosistema de investigación sobre Formación Profesional en España
}

\section{The path towards a research ecosystem on vocational education and training in Spain}

\author{
Benito Echeverría Samanes* y Pilar Martínez Clares** \\ *Universidad de Barcelona \\ **Universidad de Murcia
}

\begin{abstract}
Resumen
La mejor forma de fomentar la Investigación sobre Formación Profesional en España (IFPE) es a través de un ecosistema que conecte el potencial tecnológico, investigador, innovador y de emprendimiento con las infraestructuras y talento de sus investigadores y agentes sociales $y$ se refuerce con principios, políticas e inversiones de la estrategia a seguir. Un buen ecosistema de IFPE difícilmente se puede configurar, sin redes sólidas de conexión entre la ciencia, actores socio- económicos y la sociedad en general. Como en otros muchos campos de la actividad, las innovaciones en FP surgen de los intercambios y colaboraciones entre investigadores, centros de formación, universidades, empresas e inversores. Para alcanzar los objetivos, directa o indirectamente relacionados con la FP, ha de implicarse todos los actores /agentes: gobierno, sector privado, sociedad civil y personas particulares. Construir una cultura de investigación e innovación en FP supone un continuo proceso de reflexión-acción-reflexión, que ofrezca espacios reales de intercambio, participación y toma de decisiones sobre su importante función en el desarrollo de la FP. Probablemente éste sea la mejor vía para superar su deficiente reconocimiento social en España y lograr que se convierta en motor de las transformaciones, que necesita nuestra sociedad. Sin duda alguna, experimentar y hacer ciencia en el arte de la formación de aprendices contribuye a la capacitación del capital humano, generador de los cambios y estimula su desarrollo profesional.

Palabras clave: Formación profesional; investigación; innovación; ecosistema
\end{abstract}

Correspondencia: Pilar Martínez Clares, pmclares@um.es. Departamento de Métodos de Investigación y Diagnóstico en Educación 


\begin{abstract}
The best way to promote Research on Vocational Education and Training in Spain (IFPE in Spanish) is through an ecosystem that connects the technological, investigative, innovative and entrepreneurial potential with the infrastructures and talent of its researchers and social agents, and which is reinforced by principles, policies and investments of the strategy to follow. A good IFPE ecosystem cannot be constructed without strong networks of connection between science, socioeconomic actors and society in general. As in many other fields of activity, innovation and research in vocational training arise from exchanges and collaborations between researchers, training centres, universities, companies and investors. To achieve the objectives directly or indirectly related to vocational education and training (VET), all actors must be involved: the government, the private sector, civil society and individuals. Building a culture of research and innovation in VET involves a continuous process of reflection-action-reflection, which offers real spaces for exchange, participation and decision-making on its important role in the development of VET. This is probably the best way to overcome its poor social recognition in Spain and convert it into the engine of the transformation that our society needs. Without a doubt, experimenting and science-making in the art of training apprentices contributes to the training of human capital, the generation of change and the stimulation of their professional development.

Keywords: Vocational education and training; research; innovation; ecosystem
\end{abstract}

\title{
Introducción
}

Una de las cinco prioridades de la Unión Europea (UE) en materia de educación y formación profesional (EFP) (2015-2020) ha sido desarrollar mecanismos de garantía de calidad de la EFP, de acuerdo a la recomendación del Marco de Referencia Europeo de Garantía de la Calidad en la Educación y Formación Profesional (EQAVET). Para ello, estableció circuitos continuos de información y retroalimentación de los sistemas de Formación Profesional Inicial (FPI) y Formación Profesional Continua (FPC) como parte de la garantía de la calidad, evidenciada a través de la investigación. "Aumentar las sinergias entre las actividades de educación, investigación e innovación, con una perspectiva de crecimiento sostenible", sigue siendo una de las acciones prioritarias recabadas por el Informe conjunto del Consejo y de la Comisión (2015) sobre la aplicación del marco estratégico para la cooperación europea en el ámbito de la educación y la formación (ET 2020).

El panorama de la investigación europea sobre FP no es tan halagüeño como a primera vista puede parecer. Los últimos anuarios de International Journal for Research in Vocational Education and Training (IJRVET 2017, 2018, 2019) dejan traslucir que esta actividad investigadora se caracteriza por ser: a) Un Área de investigación relativamente joven, que comienza a abrirse camino asociada a la globalización y digitalización; b) Con investigaciones circunscritas en la mayoría de los casos a Estados concretos y algunos estudios comparativos de reducido número de países; c) Sin una clasificación normalizada de la investigación sobre FP; d) Con notables limitaciones en las fuentes de datos, que afectan a la comparabilidad de los mismos y a los resultados de las investigaciones; e) Concentrada en el área de educación con escasa presencia 
de otras disciplinas relacionadas con la FP; f) Dispersa, fragmentada y con deficiente fundamentación de las investigaciones y g) Sin líneas de investigación europeas, ni en áreas temáticas, ni en el programa de investigación "Horizonte 2020"(Moso, p.15-17).

La mayoría de estas deficiencias se observan también en España, al menos con respecto a la Formación Profesional Inicial (FPI). En general, la investigación en este subsistema es escasa, discontinua, dispersa temporal y geográficamente, con fuentes de financiación insignificantes y poca implicación en su desarrollo por parte de las universidades y entidades afines. Son exiguos los grupos de investigación consolidados con líneas de investigación asentadas sobre esta temática y prácticamente inexistentes los cauces comunicación entre los investigadores de FP. La mayoría se agrupan en el Área de Ciencias de la Educación y hay una ausencia total de investigaciones sobre empresas formadoras. Casi todas son de carácter descriptivo, con muestras reducidas y por lo general no aleatorias. Predominan como instrumentos de medida las entrevistas y cuestionarios, no siempre convenientemente validados y fiabilizados (Echeverría y Martínez-Clares, 2019a, p.85-89).

A estas conclusiones se ha llegado en dos recientes estudios realizados con el patrocinio del Centro de Conocimiento e Innovación de la Fundación Bankia por la Formación Dual y de la Fundación Bertelsmann. El primero dedicado al "Diagnóstico de la investigación sobre la Formación Profesional Inicial en España -2005/2017-“(Echeverría y Martínez-Clares, 2019a). El segundo centrado en los "Retos y estrategias de acción en torno a la investigación sobre Formación Profesional en España" (Echeverría y Martínez-Clares, 2020), ampliando el ámbito de la investigación más allá de la FP español.

\section{Propósito}

Los dos estudios emanan de la voluntad decidida de estimular un modelo de desarrollo de la FP basado en la investigación e innovación, contando con la participación de los actores implicados directa o indirectamente en su crecimiento. Una formación imbricada en la cultura científica, que busca la excelencia y calidad a través del pensamiento crítico. El resultado de esta cultura de investigación es una formación innovadora, en la que se revisa constantemente los perfiles profesionales requeridos, la planificación, organización, gestión, contenidos, metodologías, tutorías,...etc. y la función de todos los equipos de agentes implicados. Una Formación avivada por la "P" de profesional, proactiva y presente en los entornos educativos y laborales, en los procesos de búsqueda de empleo, de inserción socio laboral y de las transiciones a lo largo de la vida, así como en espacios de cohesión e inclusión social.

Ambos análisis tratan de estimular un modelo de crecimiento y desarrollo de la FP que juega un papel esencial en la integración social y de ahí la necesidad de evidencias que permitan demostrar la funcionalidad, eficiencia, eficacia de su contribución al desarrollo y equilibrio socio-económico, acorde a los Objetivos de Desarrollo Sostenible de la Agenda 2030 de Naciones Unidas.

Se pretende implementar, fomentar e incrementar la IFPE, al tiempo que informar, divulgar y difundir su potencialidad, conscientes de que lo desconocido, no existe. Se intenta, mediante vías de comunicación e impulso a la investigación e innovación 
sobre FP, reconstruir su realidad y obtener evidencias de qué se hace, cómo se hace, dónde se hace, para qué se hace y de qué forma mejorar lo que se hace, con el fin de inocular ciencia en el arte de formar aprendices (Echeverría y Martínez-Clares, 2019b). Un proceso que implica creer y crear, para hacer crecer la FP en España, a través de la difusión del conocimiento y transferencia de los resultados de la investigación. Todo ello dentro de un ecosistema de la IFPE en el que participen las administraciones públicas, el mundo académico, las empresas, los centros formativos y la sociedad en general, ya que difícilmente se puede configurar sin redes sólidas de conexión entre la ciencia, actores socio-económicos y la sociedad.

\section{Ecosistema de la IEFP}

\section{Contexto}

El Gobierno de España (2019, p.3), declaraba hace un año que “España disponía de una situación económica favorable para iniciar un proceso de reformas que aborden los retos socioeconómicos del siglo XXI". Ahora bien, tal como le recomienda la Comisión Europea, ha de aprovechar la situación económica actual, para abordar las reformas pendientes con vistas a hacer la economía española más resiliente y afianzar el crecimiento de la productividad. En concreto, se insta a mejorar los servicios sociales, el empleo y la educación, aumentar la inversión en investigación y desarrollo y promover el buen funcionamiento de los mercados y las instituciones.

En efecto, es fundamental una decidida apuesta por la formación y el capital humano, ya que "durante las últimas décadas, la economía española ha crecido de manera intensa, pero no ha sido capaz de compatibilizar la creación de empleo con mejoras sustanciales de la productividad del trabajo. Las causas de estos escasos avances se han relacionado a menudo con la calidad de la educación y la falta de inversión en capital humano, esto es, con la cualificación y la formación continua de los trabajadores" (Gobierno España, 2019a, p. 34).

"Los diagnósticos de muchos estudios recomiendan dos vías para lograr que el capital humano se traduzca en una mejora de la productividad. La primera vía consiste en mejorar las competencias educativas y frenar el fracaso escolar y el abandono hasta reducir la tasa de fracaso al $15 \%$ "... "La segunda vía es ajustar la formación a los requerimientos del mercado laboral y garantizar la formación continua, para mejorar la productividad del trabajo, evitar la exclusión laboral y mejorar la empleabilidad de los trabajadores. En este contexto, la reforma y fortalecimiento de la Formación Profesional resulta una de las líneas estratégicas a abordar en los próximos años como medio para atraer a jóvenes y reciclar a los trabajadores de forma continua en las nuevas profesiones sobre las que va a girar el crecimiento económico" (Gobierno de España, 2019a, p. 34-36). Entre el primero de estos fines cabe destacar el Plan Estratégico de Formación Profesional Dual con fórmulas flexibles y creación de consorcios, que permitan a las empresas de cada sector incorporarse al diseño de las cualificaciones y formaciones asociadas y la formación de los estudiantes y la actualización del profesorado (Gobierno España, 2019a, p. 37) 
No se trata de que la investigación sea un añadido más a las políticas de FP, sino que se convierta en el eje transversal de las mismas.

\section{Dispositivos configuradores}

En la configuración del ecosistema es preciso un enfoque inclusivo y comprensivo que facilite la participación de las administraciones públicas -estatales y autonómicas-, de los actores socio-económicos, de la academia y de la sociedad en general. La orientación de las actuaciones promovidas por la Comisión Europea, es partidaria de integrar asociaciones público-privadas, redes de investigación, mundo académico, empresas, centros formativos, etc., a través del Centro Europeo para el Desarrollo de la Formación Profesional (CEDEFOP). A un nivel más reducido, es también el planteamiento que impregna la actividad del Instituto Federal de Formación Profesional de Alemania (BIBB).

Los retos a los que se enfrenta la IFPE, difícilmente se pueden abordar desde un planteamiento exclusivamente gubernamental. Dificultaría la convergencia de prioridades, objetivos y medidas de los distintos miembros implicados en la FP, con el consiguiente perjuicio para la necesaria coordinación posterior en el desarrollo de las estrategias de acción.

España dispone del conocimiento y de los profesionales necesarios para un correcto desarrollo de la investigación en este campo, pero precisa de un buen sistema de coordinación. Necesita configurar un ecosistema, que subsane esta carencia, tanto a la hora de diseñar un plan estratégico de investigación, como en las fases de desarrollo, revisión y evaluación del mismo. Esto requiere un determinado modelo de gobernanza para gestionar vertical y horizontalmente la FP en general y la investigación sobre la misma en particular, que contemple el futuro desde la perspectiva de la estrategia, en lugar de hacerlo desde la miopía de tácticas momentáneas.

No se parte de cero, ni es cuestión de desestimar los esfuerzos realizados para mejorar el modelo. Se han de considerar las fortalezas y aprovechar oportunidades, así como considerar los avances los observados, por ejemplo, en el Consejo General de Formación Profesional (CGFP), a través del Acuerdo Estratégico por la Formación Profesional o mediante el I Plan Estratégico de Formación Profesional del Sistema Educativo 2019-2022.

Es de valorar la reciente creación de los cinco grupos de trabajo del CGFP, anunciados en el Seminario Internacional Formación para el empleo (2018), cuyos objetivos coinciden con bastantes de las temáticas de investigación prioritarias identificadas por los grupos focales de nuestro estudio sobre retos y estrategias de acción: 1) Desarrollo, evaluación y calidad de todo el sistema de FP; 2) Información y orientación profesional; 3) Centros nacionales de referencia y centros integrados de FP; 4) Formación Profesional Dual; 5) Acreditación de competencias profesionales adquiridas a través de la experiencia laboral. Bien es cierto que el CGFP es un órgano consultivo, pero en él participan las autoridades educativas y laborales responsables de la FP junto con sindicatos y asociaciones de empleadores, y puede ser un excelente medio para asentar las bases de un ecosistema de IFPE. 
Como también puede coadyuvar a mejorar el modelo de gobernanza y coordinación el Acuerdo Estratégico por la Formación Profesional, firmado poco antes (10.09.2018) entre el gobierno, la patronal y los sindicatos, para "dar un impulso estratégico rápido y urgente a todo el sistema de FP como pieza clave para garantizar la fortaleza competitiva y la mejora de la empleabilidad en España" (Pedro Sánchez, Presidente de Gobierno). Según el llamamiento a la corresponsabilidad e implicación, realizado por la Ministra de Educación y Formación Profesional -Isabel Celaá- "es imprescindible abrir un escenario de trabajo compartido para que las empresas y los agentes sociales sean protagonistas directos de una Formación Profesional más moderna e innovadora, tal y como merece este país... Un país sin una FP de calidad es un país sin futuro". Con este acuerdo se busca sobre todo agilizar el ajuste entre la oferta y la demanda de cualificaciones a corto y medio plazo, haciendo partícipes a las empresas y a los sectores productivos a través el Observatorio del Instituto Nacional de Cualificaciones (INCUAL).

Este Acuerdo comenzó a concretarse en el Plan Estratégico de Formación Profesional del Sistema Educativo 2019-2022, pero sólo parcialmente al no contemplar la Formación para el Empleo. El Ministerio de Educación y Formación Profesional manifiesta que ha contado para su elaboración con cuatro Ministerios más, entre ellos el de Trabajo, y se fija como prioridad fundamental la colaboración y participación de las empresas y la apertura a los órganos de participación de todos los sectores económicos, para responder ágilmente a las necesidades de cualificación de cada uno de ellos. Sin embargo, para el sindicato CCOO (2019) "no es una buena noticia para avanzar en la integración de la formación que se pretendía con la Ley 5/2002 de las Cualificaciones y la Formación Profesional... La exclusión de la Formación para el Empleo de este plan estratégico, parece asumir la incapacidad de ambos ministerios - Educación y Trabajo - de establecer objetivos comunes y desarrollar de manera coordinada la mencionada Ley"

A través de sus 9 ejes, 16 objetivos estratégicos y 45 líneas de actuación pretende: a) Mejorar la empleabilidad y satisfacer las necesidades del mercado laboral, mediante la renovación del Catálogo de títulos de FP; b) Consolidar la flexibilización de estas enseñanzas, aumentar la oferta formativa, mejorar la accesibilidad y el reconocimiento de competencias; c) Actualizar y perfeccionar la formación del profesorado; d) Modificar la normativa de la FP del sistema educativo; e) Internacionalizarla ; f) Apostar por la orientación profesional como un elemento imprescindible de calidad de la FP.

Son metas dignas de encomio, pero no contemplan partida presupuestaria alguna, y solo uno de los nueve ejes se refiere al "desarrollo e implementación de un sistema estatal de evaluación y calidad de las enseñanzas de Formación Profesional del sistema educativo" (Eje 9), con el intento de "diseñar un Sistema Estatal de Calidad de la FP" (Objetivo 16), a través de cuatro líneas actuación $(42,43,44,45)$ con indicadores de logro que difícilmente permitirán investigar la funcionalidad, eficiencia y eficacia de su eje noveno en particular y del I Plan Estratégico de FP en general.

Donde la investigación e innovación adquieren un papel muy predominante es, por ejemplo, en el V Plan Vasco de Formación Profesional 2019-2021. La Formación Profesional en el entorno de la $4^{a}$ Revolución Industrial. Queda patente en los primeros objetivos de casi todos sus nueve ámbitos estratégicos, pero especialmente en el tercero -"Inteligencia colaborativa y flexibilidad transformadora"- y en el noveno dedicado 
a las "Estructuras orgánicas especializadas", sobre las cuales sustentar la actividad investigadora e innovadora.

Una de las líneas de actuación del primero de estos ámbitos es la de "Desarrollar la capacidad científica e investigadora de la FP en Euskadi" a través de "1) Orientar y participar en las líneas de investigación relevantes para la FP; 2) Desarrollar vocación investigadora en las personas docentes de la FP; 3) Acceder a los programas de financiación a la investigación aplicada" (p. 50).

\section{Visión estratégica}

Es evidente que asistimos a la superación de la secular infravaloración en la que ha estado inmersa la FP en España (Echeverría, 1993, pp.79-180). Las citadas evidencias, pueden considerarse hechos aislados, pero cada vez mayor número de personas implicadas en el desarrollo de la FP concuerdan en la imperiosa necesidad de poner ciencia en el arte de formar aprendices. Ello requiere aunar esfuerzos dentro de un ecosistema que requiere la corresponsabilidad de los diferentes actores (gráfico 1).

Desde la perspectiva de esta visión estratégica, se ha conocer el estado y tendencias de la investigación en este campo tanto fuera como dentro del ecosistema español, con el fin de aquilatar sus objetivos, los medios y las acciones necesarias para su desarrollo, al tiempo que se establecen las prioridades de respuesta a los retos de investigación sobre FP. Es una labor pendiente de realización en España, ya que ni la Administración General del Estado, ni las Autonómicas, ni mucho menos las locales, acaban de implicarse seriamente en este quehacer.

La articulación del ecosistema ha de arrancar con una evaluación objetiva del estado de la IFPE - de dónde venimos, dónde estamos y hacia dónde nos dirigimos-, para superar la visión limitada y fragmentada ofrecida por las valoraciones existentes.

Se precisa información válida, fiable, comprehensiva y sustentada en evidencias, para evitar el riesgo de que objetivos, prioridades o líneas de actuación no se asienten sobre sólidos puntos de partida científicos y contrastables. Como en otros muchos campos de investigación, el ecosistema debería disponer de un mapa de la IFPE y de un índice periódico, dinámico y público - un "Libro Blanco de la IFPE", de cuya elaboración puede encargarse algún centro de investigación e innovación similar al BIBB, $\mathrm{u}$ otras entidades existentes, como el CGFP o el INCUAL de quien depende.

A partir del mejor conocimiento posible del estado de la IFPE, se deberá proceder a un análisis estratégico que permita identificar y seleccionar los nichos y las actividades esenciales, donde aplicar la investigación con mayor impacto sobre las metas previstas. El alineamiento de las prioridades investigadoras, la inversión y el impulso de los miembros del ecosistema reducirá la dispersión actual de objetivos y estrategias públicas y privadas, así como el riesgo de que los esfuerzos en IFPE se agoten en la obtención de rendimientos a corto término y no se emprendan líneas de actuación a largo plazo. 


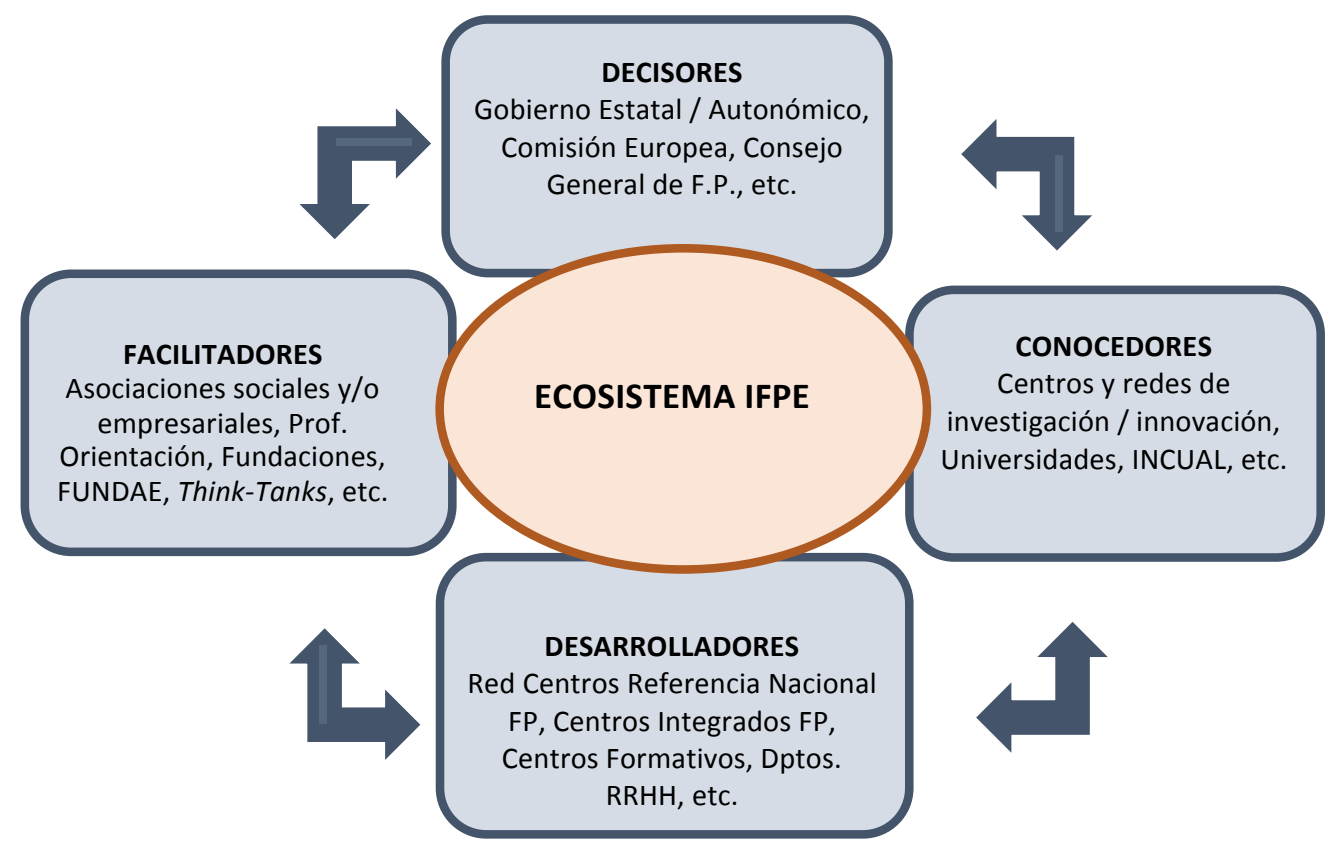

Gráfico 1. Ecosistema de la Investigación sobre la Formación Profesional de España (IFPE)

Son muchas las problemáticas a investigar, pero no sólo ha de atraer la atención las del momento presente, sino que además se ha de estar atento a los signos de los tiempos, explorar las necesidades futuras y buscar respuestas, aplicando más innovación al ámbito de la investigación y aumentando la cooperación entre los mundos de la ciencia y de las empresa, para orientar la actividad de I+D+I a resultados que contribuyan a la creación de empleo y riqueza económica y bienestar social.

Como se plasma en el V Plan Vasco de FP, el ecosistema de IFPE ha de tener muy en cuenta "las estrategias de investigación e innovación para la especialización inteligente - Research and Innovation Smart Specialisation Strategy (RIS3) -, que forman parte de las distintas estrategias globales impulsadas por la Unión Europea: Estrategia Europa 2020, Horizonte 2020, Unión por la Innovación, la Estrategia Europea relativa a las Tecnologías Facilitadoras Esenciales y el Marco Estratégico Común desarrollado para la aplicación de los Fondos Estructurales" (p. 30).

Ha de procurar integrar capacidades de conocimiento con capacidades productivas, generando transferencias entre ambas, lo que implica notables cambios de mentalidad en las administraciones públicas, en las empresas y en la sociedad en general. Ha de vencerse la resistencia de las primeras a superar la ancestral separación de responsabilidades en torno a la FP entre las administraciones de educación y trabajo, pero también con las de ciencia, economía, industria, etc., tanto a nivel estatal como autonómico. No serán menores los esfuerzos para superar la renuencia de las empresas a implicarse en el desarrollo de la FP en general y de la IFPE en particular, como recientemente 
está quedando patente, por ejemplo, en el proceso de implantación de la Formación Profesional Dual en España (Echeverría, 2016 y Echeverría y Martínez-Clares, 2018a). Y aún más ardua es la labor con respecto a la sociedad en general y a las familias en particular.

"Es fundamental aprovechar sinergias, reordenar y optimizar recursos y coordinar servicios de orientación, tanto del ámbito educativo como del empleo. Desde ambos entornos se ha de procurar aunar criterios para potenciar un Sistema Integrado de Información y Orientación, que reconsidere el nuevo concepto de orientación permanente" (Echeverría y Martínez-Clares, 2015, p. 3).

Al igual que es esencial potenciar el debate sobre las temáticas y enfoques metodológicas de la investigación sobre orientación (Bosada, 2019), dado el rol estratégico ejercido por la intervención orientadora, reconocido por organismos tales como CEDEFOP, Comisión Europea, ETF, OCDE, OIT y UNESCO en el informe Investing in Career Guidance (CEDEFOP 2019a) y corroborado por numerosas investigaciones, relacionadas incluso con la validación de aprendizajes no formales e informales (CEDEFOP 2019b).

En síntesis, es preciso fomentar una cultura transformadora entre los responsables públicos y privados, para lo cual el ecosistema IFPE -mutante, regenerador y adaptable- ha de contar con el liderazgo del Gobierno, de las empresas, de las universidades y de centros de investigación e innovación.

\section{Recursos humanos y materiales}

Una de las grandes dificultades para el desarrollo de IFPE es la disponibilidad de equipos de investigación asentados, con autonomía y capacidad de transferir conocimiento a las administraciones, a las empresas, a los centros formativos y a la sociedad en general, al igual que ocurre en otros campos del saber (Mato, 2018).

Es bien conocido que el contexto laboral de los investigadores de nuestro país es precario, con bajos salarios y escasas oportunidades de desarrollo profesional. Por ello, una de las prioridades de este ecosistema ha de ser la de atraer, retener y rescatar talento, mediante solventes y reputados entornos laborales e incentivos económicos, que ofrezcan estabilidad laboral y expectativas de promoción profesional.

Para subsanar las deficiencias existentes, se necesitan perfiles investigadores multidisciplinares, configurados a partir de materias curriculares de grados y posgrados relacionados con la $\mathrm{FP}$, de masters especializados en la misma y sobre todo de programas de doctorado, a poder ser interuniversitarios. Igualmente, se precisan cambios formativos en las administraciones públicas, para dotar a los funcionarios de conocimientos y cultura de investigación, así como en las empresas, para sensibilizar a directivos y cuadros intermedios sobre la misma. En un ecosistema como el propuesto, administraciones públicas, universidades, empresas y centros de investigación pueden compartir recursos humanos y retribuir mejor a los investigadores, ahorrándose gastos de intermediación, que con frecuencia van a parar a consultorías o servicios similares. 
Una buena manera de comenzar a articular el ecosistema podría ser mediante hubs, generadores de sinergias e impulsores de proyectos de Innovación, que busquen soluciones a los problemas más acuciantes de la FP a través de informes, encuentros, talleres, etc. Lo ideal sería que contasen con financiación del sector privado - fundaciones o similares -, para hacer confluir y arraigar una masa crítica de investigadores de reconocida solvencia, junto a recursos gubernamentales, que orienten el ecosistema hacia aquellos servicios públicos donde la IFPE está llamada a jugar un papel esencial. Simultáneamente o a posteriori, podrían agregarse bienes de diferentes administraciones públicas, grandes empresas, agrupaciones sociales e incluso contar con la contribución de algunas pymes, dada la configuración del tejido empresarial español.

Esta articulación facilitaría a medio o largo plazo la existencia de un centro de excelencia, que bien podría ser en red, donde compartir talento y financiación, añadiendo valor al ecosistema de IFPE. De esta forma los investigadores podrían estar mejor retribuidos, sin tener que renunciar a su actividad habitual, ni al lugar de residencia, contribuyendo al mismo tiempo tanto al desarrollo teórico como práctico de la FP. El objetivo último es contar con grupos de investigadores dedicados a determinados campos de la IFPE en sus respectivas áreas de especialización. Así, los hubs pueden servir para captar, retener o recuperar talento, además de facilitar la participación en redes de excelencia y el intercambio de investigadores, públicos o privados.

A la dificultad de disponer de talento necesario, se une el problema de la inversión e inversores. Como ocurre en otros ecosistemas, "la financiación es el talón de Aquiles de las estrategias porque las agendas y planes para su desarrollo carecen de seguridad y estabilidad en los marcos financieros disponibles. No suelen disponer de programas plurianuales ni rebasar el marco de las legislaturas, por lo que se deciden año a año en función de la coyuntura y generan imprevisibilidad en situaciones económicas normales e inestabilidad en situaciones difíciles" (Arteaga y Ortega, 2019, p.29). Sin partidas presupuestarias para la IFPE, es difícil que la sociedad española tome conciencia de la importancia de la IFPE para su prosperidad y bienestar.

El sector privado no puede esperar que el sector público invierta en la IFPE, que precisan los intereses particulares de sus negocios. Tampoco el sector público puede confiar en que el sector privado va a atender intereses que están fuera del mercado. Entre los intereses específicos de uno y otro sector se puede encontrar una zona común entre ambos, que permita compartir inversiones y riesgos, para diversificar y estabilizar la financiación. La corresponsabilidad del ecosistema lo facilita.

Podría aumentar la inversión en IFPE, si se incentiva fiscalmente a las empresas, para que participen en proyectos financiados con dinero público. Sobre todo, flexibilizando el control previo, tal como sucede con la propia Comisión Europea, a base de una gestión rigurosa pero no agobiante, como es el caso con la I+D. De igual modo se podría acrecentar la inversión, si se aprovechan algunas oportunidades de financiación de la Unión Europea, si bien el ecosistema deberá disponer de cierta autonomía financiera, para participar en los proyectos.

A todo ello puede contribuir la creación de un portal donde publicitar proyectos de investigación, que facilite la conexión de los interesados (proyecto/datos/resultados) e incentive la participación en proyectos europeos con subvenciones, aportando, por ejemplo, personal que ayude a gestionar estas convocatorias. 


\section{Gobernanza}

Cada ecosistema necesita un modelo de gobernanza construido a medida, aunque algunos pueden servir de referente para el de IFPE, como, por ejemplo, el propuesto por el Real Instituto Elcano para la Inteligencia Artificial (Arteaga y Ortega, 2019). No es cuestión de copiar modelos, porque cada ecosistema ha de experimentar su propia estructura y dinámica, pero sí de reconsiderar tendencias, metodologías de trabajo y prácticas innovadoras funcionales, eficientes y eficaces.

Este contraste deja patente el poco sentido que tiene esperar a diseñar un modelo ideal, para ponerlo en funcionamiento. Cada ecosistema es diferente y se perfecciona por ensayo y error. Su desarrollo ha de articularse gradualmente a partir de lo que funciona, mediante proyectos piloto que sirvan para estimular el mejor funcionamiento posible.

Lo que sí es ineludible es el carácter inclusivo del ecosistema. No puede relegar a nadie que pueda contribuir a su desarrollo y esté dispuesto a hacerlo. Eso no quiere decir que todos lo hagan de la misma forma. La contribución ha de ser asimétrica, para facilitar la aportación de todos los miembros, desde los aprendices y sus entornos más cercanos, hasta los investigadores y los políticos directa o indirectamente implicados en la IFPE. Y lógicamente la responsabilidad en las decisiones no será la misma de unos que de otros.

Es evidente que la responsabilidad de las decisiones relacionadas con la FP en general y la IFPE en particular está excesivamente fragmentada en la Administración General del Estado. No es cuestión tanto de centralizar las competencias, ni siquiera de crear un Ministerio como el actual de Educación y Formación Profesional, si no de racionalizarlas, integrando las responsabilidades de FP que actualmente están dispersas por diferentes Ministerios y Consejerías.

Algo similar ocurre con la cooperación dentro del sector privado y entre ambos sectores, por lo que el ecosistema ha de concebir su propia dinámica de interacción, como también se requiere en el de la cooperación pública - privada. Con el ecosistema en red, el sector público ya no tiene por qué controlar la iniciativa y el proceso de decisiones en torno a la IFPE, como acostumbra tradicionalmente.

Sin una estructura jerárquica las iniciativas y decisiones emanan y se comparten por diferentes canales de manera mucho más ágil y flexible, facilitando la agrupación de esfuerzos investigadores por afinidad de intereses (plataformas). El carácter distributivo del ecosistema favorece el flujo de las decisiones y la conexión entre los actores de los diferentes niveles del ecosistema (gráfico 1) y su relación fluida con los de otros ecosistemas.

Eso sí, dada la naturaleza del ecosistema propuesto, han de delimitarse claramente los diferentes niveles de responsabilidades y los elementos configuradores de los mismos, esbozados a continuación.

\section{A) Nivel estratégico}

El ecosistema de IFPE ha de contar con una vigorosa y dinámica inteligencia estratégica, muy atenta a las grandes transformaciones tecnológicas, económicas y sociales 
(Echeverría et. alt. 2008), predecesoras de la Revolución 4.0 (Echeverría y MartínezClares, 2018b) y de la quinta (Vidal, 2019), ya que está llamada a ejercer su liderazgo, fijando las prioridades del ecosistema, sobre todo desde:

- Presidencia -alto comisionado o similar-, asistido por un equipo asesor de dirección, representativo del ecosistema, que ayude a formular y supervisar planes y políticas a desarrollar.

- Consejo de autoridades públicas y privadas, encargado de adoptar directrices genéricas (estrategias, planes de acción), que deberá contar también con el respaldo y participación de la de sociedad civil, dado el carácter inclusivo del mecanismo de gobernanza.

\section{B) Nivel operativo}

Es el responsable de que el ecosistema gestione las directrices emanadas del nivel estratégico, contando para ello con:

- Consejo de dirección (representantes ejecutivos de las autoridades del ecosistema).

- Agencia/Oficina/Unidad autónoma de gestión, de carácter técnico, donde confluyan los recursos necesarios, que garanticen el desarrollo y continuidad de la IFPE.

- Foro de Participación: Órgano asesor del Consejo con participación social, de dentro y fuera del ecosistema, para asegurar su conexión con la realidad social, sindical, política, etc.

- Grupo de concienciadores y dinamizadores culturales que promuevan el acercamiento de empresarios, sindicatos, formadores, profesionales de la orientación, etc. a la IFPE.

\section{C) Nivel táctico}

Elementos que posibilitan la ejecución de iniciativas y actividades del ecosistema, tales como:

- Centro de investigación e innovación, donde se agrupen recursos humanos y materiales

- Plataforma de IFPE: Ventanilla única para acceder a los recursos que facilita el ecosistema.

- Hub de IFPE.

- Red estatal de investigadores que fomente el desarrollo y consolidación de grupos de investigación interdisciplinarios, conectados a redes europeas.

- Infraestructuras nacionales (bases de datos, microdatos, etc.), europeas e internacionales 


\section{Información y comunicación}

Podemos contar con un excelente modelo de gobernanza, pero de poco servirá, si no se logra transmitir el trascendente papel de la FP en el desarrollo y bienestar de una sociedad como la nuestra, un tanto reacia a valorarla y aún más asumir la función esencial de la investigación en su mejora.

En primer lugar, es preciso concienciar y hacer comprensible a los políticos, docentes de todos los niveles educativos, profesionales de la orientación, empresarios, sindicatos y a la ciudadanía en general el importante papel que la educación en general y la FP en particular está llamada a jugar en la cuarta y quinta revolución industrial y por ende la IFPE. Nada mejor para tal fin, que aprovechar los aires a favor de ella en la UE y los que están emergiendo en España, hasta lograr que el ecosistema logre comunicar un mensaje ilusionante a la sociedad en torno a la IFPE. Para ello, no puede perderse de vista el contexto relacional, si desea conseguir la óptima participación en las metas perseguidas y la necesaria comunicación interactiva.

En segundo lugar, al relato informativo ha de acompañar la elaboración de una reputación solvente de marca, acoplando la capacidad científica del ecosistema con una gestión eficiente y eficaz, un fuerte liderazgo y el imprescindible apoyo político y social. Esta reputación solvente es la que permitirá atraer talento e inversiones con el respaldo de entidades punteras que a su vez contribuyan a lograr la misma.

En tercer lugar, el relato y la reputación sin duda contribuirán a lograr que investigadores, gestores, desarrolladores, facilitadores y usuarios se identifiquen con el ecosistema. Su actividad en entornos de trabajo compartidos facilitará el desarrollo de una comunidad epistemológica en torno a la IFPE, con el suficiente prestigio para sentirse orgullosos de pertenecer a la misma y para atraer savia nueva, siempre que genere la suficiente confianza mediante la puesta en funcionamiento de normas y mecanismos de evaluación, verificación y validación.

La comunicación, el relato y la reputación son a su vez fundamentales para impulsar las relaciones del ecosistema de IFPE con investigadores del Centro Europeo para el Desarrollo de la Formación Profesional (CEDEFOP) o de redes como VETNET, perteneciente a la Asociación Europea de Investigación Educativa (EERA), apoyada por la Red Internacional de Investigación en Educación y Formación Profesional (IRNVET) e integrada en la Asociación Mundial de Investigación en Educación (WERA) (Nägele \& Stalder, 2018).

\section{Conclusiones}

En pleno S. XXI es necesario disponer de un ecosistema de la IFPE de estas características, ya que la investigación es un motor de desarrollo y equidad de gran poder transformado, que permite encontrar nuevas respuestas, más eficaces y eficientes, a los principales desafíos de la sociedad. Es la base para crear conocimiento, avanzar y lograr a través de ella una FP innovadora, que responda a las necesidades y optimice sus procesos.

Ello requiere un compromiso con la investigación e innovación en FP y extender su valor e impacto, para que actúen como catalizadoras de la transformación de este 
sistema formativo. No podemos conformamos con una FP a espaldas de ellas. Sería como ir a contracorriente no solo de las tendencias más progresivas del entorno europeo y sino también de los Objetivos de Desarrollo Sostenible de Naciones Unidas. La investigación e innovación son claves para la acción transformadora de la FP.

Una FP asentada sobre la investigación, es una formación de presente y de futuro, que forma parte de una cultura científica, que mediante el pensamiento crítico actúa responsablemente en la búsqueda de excelencia y calidad. Esta cultura debe entenderse como una reconceptualización de la propia FP, a la luz de sus competencias, iniciativas, formas de gestión, normativa...etc. Ello supone un mayor compromiso con la sociedad y la contribución a un modelo de desarrollo equilibrado y sostenible, para lo cual se precisa la colaboración de todos los implicados. La investigación debe mantenerse en permanente retroalimentación con la sociedad, de modo que implique a todos los agentes/actores relacionados con la FP, marque tendencias y muestre, con el peso del saber y la práctica, lo que se ha de hacer para promover una actitud investigadora en la FP. Esta retroalimentación debe ser bidireccional y recíproca, pero es casi imposible lograr una FP investigadora en una sociedad que no invierte en investigación social.

La importancia de la investigación e innovación radica en que son un instrumento clave para el progreso de la FP. Ambas la ponen en valor, sirven para expandir su conocimiento, dan utilidad al mismo, estimulan el pensamiento crítico, muestran evidencias, combaten la desinformación ofrecen comprensión, predicción y prevención y ayudan a la toma de decisiones. A la vez, exploran la historia y mejoran su calidad, reconociendo el pasado y corrigiendo errores, favoreciendo el progreso y los nuevos avances con el fin último de mejorar y optimizar procesos. De ahí la necesidad e importancia de adquirir un compromiso con la investigación e innovación en FP, para que ésta pueda crecer, asumiendo que "el compromiso es un acto, no una palabra" (Jean-Paul Sartre)

Más que nunca, se necesita conocimiento, investigación, innovación y transferencia, dentro de un ecosistema de IFPE, que potencie su desarrollo en sus diferentes ámbitos de incidencia de la FP, ya que lo que no se conoce, lo que no se investiga, no existe.

\section{Referencias}

Arteaga, F. y Ortega, A. (2019). Hacia un ecosistema español de Inteligencia Artificial: una propuesta. Madrid: Real Instituto Elcano.

Bosada, M. (2019, 31 de enero). Cómo transferir el conocimiento de la investigación en orientación [Sitio Web]. Recuperado de https://www.educaweb.com/noticia/2019/01/31/ como-transferir-conocimiento-investigacion-orientacion-18694/

CEDEFOP (2019a). Investing in Career Guidance. Luxembourg: Publications Office of the European Union.

CEDEFOP (2019b). Coordinating guidance and validation. Luxembourg: Publications Office of the European Union.

Comisión Europea (s. f.). Política de la UE en materia de educación y formación profesional (EFP) [Sitio Web]. Recuperado de https://ec.europa.eu/education/policies/eu-policyin-the-field-of-vocational-education-and-training-vet_es 
Comisión Europea (2015). Aplicación del marco estratégico para la cooperación europea en el ámbito de la educación y la formación. Recuperado de https://eur-lex.europa. eu/legal-content/ES/TXT/PDF/?uri=CELEX:52015XG1215(02)\&from=EN

Confederación Sindical de Comisiones Obreras (2019, 29 de noviembre). La exclusión de la formación para el empleo del I Plan estratégico del FP del sistema educativo es una mala noticia para CCOO [Sitio Web]. Recuperado de https://www.ccoo.es/noticia:408208--

Echeverría, B. (1993). Formación Profesional. Guía para el seguimiento de su evolución. Barcelona: Promociones y Publicaciones Universitarias.

Echeverría, B. (2008). Configuración de la profesionalidad. En B. Echeverría (Coord.), Orientación profesional (pp. 23-68). Barcelona: Editorial UOC.

Echeverría, B. (2016). Transferencia del Sistema de FP Dual a España. Revista de Investigación Educativa, 34(2), 295-314. doi: 10.6018/rie.34.2.249341

Echeverría, B. y Martínez-Clares, P. (2015). Sistema Integrado de Orientación. Revista Electrónica Interuniversitaria de Formación del Profesorado, 18(2), 1-3. Recuperado de https://revistas.um.es/reifop/issue/view/13601

Echeverría, B. y Martínez-Clares, P. (2018a). Estrategias de mejora en la implantación de la Formación Profesional Dual en España. EKONOMIAK. Revista Vasca de Economía, 94, 178-203. Recuperado de https://dialnet.unirioja.es/servlet/articulo?codigo $=6649787$

Echeverría, B. y Martínez-Clares, P. (2018b). Revolución 4.0, Competencias, Educación y Orientación. Revista Digital de Investigación en Docencia Universitaria, 12(2), 4-34. Recuperado de http://www.scielo.org.pe/pdf/ridu/v12n2/a02v12n2.pdf

Echeverría, B. y Martínez-Clares, P. (2019a). Diagnóstico de la investigación sobre la Formación Profesional Inicial en España (2005-2017). Madrid: Fundación Bankia por la Formación Dual. Recuperado de https://www.dualizabankia.com/es/centro-de-conocimiento/ publicaciones/

Echeverría, B. y Martínez-Clares, P. (2019b, 29 de octubre). Formación Profesional, investigación y orientación [Sitio Web]. Recuperado de https://www.educaweb.com/noticia/2019/10/29/formacion-profesional-investigacion-orientacion-18961/

Echeverría, B. y Martínez-Clares, P. (2020). Retos y estrategias de acción en torno a la investigación sobre Formación Profesional en España. Madrid: Fundación Bankia por la Formación Dual. Recuperado de https://www.dualizabankia.com/es/centro-deconocimiento/publicaciones/

FUNDAE, SEPE, OIT (2018) Seminario Internacional Formación para el empleo, un factor clave en el futuro del trabajo (26-28.11.2018)

Gobierno de España (2018). Acuerdo Estratégico por la Formación Profesional. Recuperado de https://www.lamoncloa.gob.es/presidente/actividades/ Paginas/2018/100918sanchezfp.aspx

Gobierno de España (2019a). La Agenda del Cambio. Hacia una economía inclusiva y sostenible. Recuperado de https://www.mineco.gob.es/stfls/mineco/ministerio/ficheros/190208_agenda_del_cambio.pdf

Gobierno de España (2019b). I Plan Estratégico de Formación Profesional del Sistema Educativo (2012-2022) Recuperado de https://www.lamoncloa.gob.es/consejodeministros/ Paginas/enlaces/221119-fp.aspx 
Gobierno Vasco (2019). V Plan Vasco de Formación Profesional 2019-2021. La Formación Profesional en el entorno de la $4^{a}$ Revolución Industrial. Recuperado de http://www.euskadi. eus/contenidos/informacion/fpgeneral/es_def/adjuntos/V-PLAN-FP-CASazk.pdf

IJRVET (2017). Yearbook 2017. Recuperado de https://journals.sub.uni-hamburg.de/hup2/ public/site/pdfs/IJRVET_2018_Online.pdf

IJRVET (2018). Yearbook 2018. Recuperado de https://journals.sub.uni-hamburg.de/hup2/ public/site/pdfs/IJRVET_Yearbook_2019_Online.pdf

IJRVET (2019). Yearbook 2019. Recuperado de https://vetnetsite.files.wordpress. com/2020/01/ijrvet_2019_yearbook.pdf

Ley Orgánica 5/2002, de 19 de junio, de las Cualificaciones y de la Formación Profesional. Boletín Oficial del Estado, 147, 20 de junio de 2002, pp. 22437-22442.

Mato, S. [Coord.] (2018). Transferencia del conocimiento. Nuevo modelo para su prestigio e impulso. Madrid: CRUE Universidades Españolas y Santander Universidades. Recuperado de http://investigacion.ucam.edu/noticias/transferencia-del-conocimientonuevo-modelo-para-su-prestigio-e-impulso-el-nuevo-informe

Ministerio de Educación y Formación Profesional (2019). I Plan Estratégico. Formación Profesional del Sistema Educativo 2019-2020. Recuperado de https://epale.ec.europa. eu/es/content/i-plan-estrategico-de-formacion-profesional-del-sistema-educativo

Moso, M. (2019). Una aproximación a la investigación europea sobre la Formación Profesional. En B. Echeverría y P. Martínez. Diagnóstico de la investigación sobre la Formación Profesional Inicial en España (pp. 15-29). Madrid: Fundación Bankia por la Formación Dual.

Nägele, C. y Stalder, B. E. [Eds.] (2018). Trends in vocational education and training research. Proceedings of the European Conference on Educational Research (ECER), Vocational Education and Training Network (VETNET). Berna, Suiza: VETNET.

ONU (2015). Objetivos de Desarrollo Sostenible. Recuperado de https:/www.un.org/ sustainabledevelopment/es/objetivos-de-desarrollo-sostenible/

Vidal, M. (2019). La era de la humanidad. Hacia la quinta revolución industrial. Barcelona: Ediciones Deusto.

Fecha de recepción: 27 de abril de 2020.

Fecha de revisión: 6 de mayo de 2020.

Fecha de aceptación: 10 de mayo de 2020. 